\title{
Tree Based Energy Balancing Routing Protocol by Self Organizing in Wireless Sensor Networks
}

\author{
Syed Umar, P.V.R.D Prasada Rao, Sridevi Gutta \\ Dept of CSE, K L University, Guntur, AP, India
}

\begin{tabular}{l}
\hline Article Info \\
\hline Article history: \\
Received Apr 27, 2015 \\
Revised Aug 30, 2015 \\
Accepted Sep 17, 2015 \\
\hline
\end{tabular}

\section{Keyword:}

Network lifetime

Routing protocols

Wireless sensor networks

Wireless technology

\begin{abstract}
Today the wireless sensor networks (WSN) play a crucial role in wireless technology in various domains like military, medicine, communications etc. The energy conservation is the crucial factor in the WSN. The WSN is a system which has more number of nodes in which various sensors are fabricated on the nodes to monitor various factors of the given task. These nodes will form a network by connecting the one to other for the effective communication between the nodes, and sends the whole information to the base station (BS). As the nodes which we use for the WSN are of low cost and are battery operated. The main drawback is replacement of the battery in the WSN. The main goal is to conserve the energy consumption in WSN and also to balance the load on WSN. For this many protocols are designed like LEACH, PEGASIS, PEDAP, etc. in those balancing the load and time delayed. some drawbacks are there. So we proposed a protocol so called "Tree Based Energy Balancing routing Protocol by Self Organizing" (TEBRSO), in which instead of routing tables a routing tree will be used for routing from nodes to base station (BS), which chooses one root/control node for the broadcasting messages to the selected sensor nodes. By this protocol we can save the energy consumption in WSN and can extend the life time of it. The performance of this protocol is better when we compare with other energy saving protocols.
\end{abstract}

Copyright (c) 2015 Institute of Advanced Engineering and Science. All rights reserved.

\section{Corresponding Author:}

G. Siva Nageswara Rao,

Department of Computer Science and Engineering,

K L University,

Greenfields, Vaddeswaram, Guntur District, Andhra Pradesh 522502.

Email: sivanags@kluniversity.in

\section{INTRODUCTION}

Now a day the wireless Sensor Networks took a great revolution in the communications technology. In the WSN the micro sensors and micro systems are embedded which consume less power and energy. To embed the micro sensors we use the advances technology of MEMS (Micro Electrical Mechanical System) [1]. By using these types we have many advantages like fault tolerant, building a large network within seconds with effective communications [2] from the various sensor nodes. The WSN used in many domains like environment monitoring, pollution monitoring etc., the working procedure of the WSN is the nodes will collect the all the information in a huge amount it sends that to the data base which is called the base station and then the application will be executed. As we know that the WSN nodes are spread randomly and deployed in various places of the target region. If we use the macro components deployment of nodes will be difficult. To work properly we have to maintain the sufficient battery when the nodes are deployed in the target region. As the nodes are deployed in densely the nodes produce the reluctant data and these data should be combined and should reduce the transmission bandwidth. To avoid the duplication of the data we 
have many protocols and to maintain the energy consumption we have many protocols like PEGASIS, TBC, LEACH, HEED etc. Here in this we consider that we maintain some control or parent nodes which have child nodes, the information from the child nodes will be sent to the parent/ control nodes from that nodes the information will pass to the base station.

The power consumption in the nodes used for the many operations which will be helpful and some are not helpful. To avoid those unhelpful operations we propose a protocol called "Tree Based Energy Balancing routing Protocol by Self Organizing" (TEBRSO). Here the information will be collected by the nodes and it will be sent to the Base Station (BS). The network life time can be defined in many ways like 1). The time taken to start the network operation till to death of the first node. 2). The time taken to start the network operation till to death of last nodes. Here for the usage we adopt the first option. For the data fusion we consider two cases in the network.

Case I: How much the volume of data can be sent or received from its child nodes to any of the sensor nodes does not matter.

Case II: The data sent or received from the child nodes can't be fused and the message length is the sum of the own collected information and the received data.

\section{RESEARCH METHOD}

As we know the working procedure of WSN is collecting various information of the given task related and sending to the Base Station [1]. The nodes are spread in the target area deployed randomly so, the base station will be far away from the nodes after finishing the task the nodes will die due to the more energy consumption. To solve such energy inefficiency many protocols was proposed, some of them are LEACH, PEGASIS, HEED etc. In the LEACH protocol some nodes are chosen as the cluster head or control nodes where some child nodes will be connected to those and these control nodes will act as the parent nodes, all the information of the child nodes will be collected by the cluster heads and through the cluster heads the information sent the base station. Here the energy consumption will be more so misbalancing the energy consumption is done due to more load on the nodes. In the HEED Protocol i.e., Hybrid energy efficient distributed clustering algorithm. The HEED is the improvised version of the LEACH in selecting the Cluster head $\mathrm{CH}$. At each time HEED protocols selects the $\mathrm{CH}$ based on the residual energy usage at the nodes. When we compare with HEED and LEACH, HEED will effectively prolongs the network life time and the drawback is consumption of energy is more.

To overcome the drawback of the HEED, PEGASIS [2] Protocols was proposed. To use the optimized power and this can be done, here the Greedy Algorithm can be used to form a chain model of the sensor nodes. This PEGASIS protocol is more power efficient protocol than the other protocols like LEACH. When we compare it with the PEGASIS we can see the PEGASIS is $300 \%$ more efficient than the LEACH. We have some other protocols like TBC (Tree Based Clustering) and PEDAP are other protocols which uses the tree based routing process. The TBC is similar to LEACH [3] and is somewhat improvised. The improvising of the TBC that with the LEACH is in which each node records the information of its neighbors and build topography through computing. The drawback is that some nodes consumes more energy which are away from the base station. The PEDAP is similar to the PEGASIS protocol and is of improvised version. [9] The drawback of this protocol build the topology which will cause the large amount of waste of energy. The $\mathrm{BS}$ in the network needs to build the topography to collect the information of the sensor nodes of parent and child nodes, so delay will be there. To overcome all the drawbacks we proposed a protocol called "Tree Based Energy Balancing routing Protocol by Self Organizing” (TEBRSO).

\section{SYSTEM MODEL}

To perform the working of this protocol we assume the following properties to design the system model to the protocol

1) The Sensor Nodes are deployed in the targeted region of square field where there will be only base station which is far away from the area and each nodes will have a unique id (identifier).

2) After the deployment of sensor nodes they will keep operate till the energy will be exhausted and the nodes are stationary.

3) Each of the node will have their own capabilities and can change the power levels and can directly communicate with the BS

4) The BS is not energy constrained and it is stationary.

5) The Sensor node can get the location information through the GPS or by the position algorithms and they are location aware. 


\section{TREE BASED ENERGY BALANCING ROUTING PROTOCOL BY SELF ORGANIZING}

The main of "Tree Based Energy Balancing routing Protocol by Self Organizing" (TEBRSO) [2]-[4] is achieve network life time longer while using for various applications. In this case at each round the Base Station assigns one root head node and broadcast its node unique id to all the coordinates of the sensor nodes to trace the path from the base station to the root nodes and from root nodes to child nodes which can be built for each nodes. In this TEBRSO the root can be changed and reconstruct routing path with less delay and using the less energy. So, by using this protocol there will be a better load balance is achieved when compared with the other protocols.

The working procedure can be classified into the following stages:

1) Initialization Stage.

2) Construction of tree path state

3) Transmission of the collected data from self-organized nodes

Exchange of data from BS to Nodes

1) Initialization State

In this stage the Base Station sends the all packets to all nodes in the network like to inform the nodes to begin the task. Here we consider the nodes as $\mathrm{N}$ to calculate the energy level of the node they use the formula.

$$
\mathrm{EL}(\mathrm{i})=\lceil\text { residual_Energy }(\mathrm{i}) / \alpha\rceil
$$

EL is the energy factor for the load balance and $\alpha$ is a constant which reflects the minimum energy unit.

Each node sends its packet to the nodes and draw a circle with Rc in the one time slot. Then all the nodes monitor the channel during this timeslot, and records all the information and stores in its memory. The memory contains the information of the neighbor nodes.

After the initial phase the "Tree Based Energy Balancing routing Potocol by Self Organizing" (TEBRSO) operates in rounds. The DATA_PAK will be send to the base station from nodes so each round contains three phases like Tree Construction phase, self organization and data collected from all the nodes and transmitted phases

2) Construction of tree path stage

In the construction phase we build a routing tree for broadcast the message, we assign a node to base station as root and broadcast root ID and root and the network life time of various protocols are shown below Table 1. Each node tries to selects a control node/ main node which have nodes neighbors using the EL [5].

Table 1. Lifetime of Network of Different Schemes

\begin{tabular}{|c|c|c|c|}
\hline $\begin{array}{c}\text { Energy } \\
\text { (J/node) }\end{array}$ & Protocol & $\begin{array}{c}\text { The round a node } \\
\text { begins to die }\end{array}$ & $\begin{array}{c}\text { The round all the } \\
\text { nodes are dead }\end{array}$ \\
\hline \multirow{4}{*}{$\mathbf{0 . 2 5}$} & LEACH & 118 & 243 \\
\cline { 2 - 4 } & PEGASIS & 246 & 568 \\
\cline { 2 - 4 } & TREEPSI & 267 & 611 \\
\cline { 2 - 4 } & TBC & 328 & 629 \\
\cline { 2 - 4 } & GSTEB & 389 & 677 \\
\hline \multirow{4}{*}{$\mathbf{0 . 5}$} & LEACH & 209 & 435 \\
\cline { 2 - 4 } & PEGASIS & 485 & 1067 \\
\cline { 2 - 4 } & TREEPSI & 532 & 1123 \\
\cline { 2 - 4 } & TBC & 589 & 1165 \\
\cline { 2 - 4 } & GSTEB & 730 & 1330 \\
\hline
\end{tabular}

Every node chooses one main/control node from its neighbor nodes and every node records its neighbor's information in the form of table. By that we can know clearly which node acts as parent node and which node acts as child node and we can compute the EL. If the parent node has no child then itself acts as leaf node to it.

Each node sent a every packet to its parent nodes will be fused with the consumption of minimum energy can be chose by node nearest to it. "Tree Based Energy Balancing routing Protocol by Self Organizing" (TEBRSO) by this approach a routing tree is constructed and the nodes will be still have the possibility of connecting to their nearest neighbors. To construct the routing tree we use the BS to computes 
the topography Even though we can fulfill this work without the control of BS, a large amount of energy is wasted in the next phase

3) Transmission of the collected data from self-organized nodes [2], [3]

After the completion of the Initialization stage, Tree construction for the routing purpose and we go for the transmission of data from BS to nodes and after the given task completed the nodes sends the information to Base Station for the required analysis. Here the each node collects the information to generate the DATA_PAK which is transmitted to Base Station [7]. The Transmission process can be explained in three segments below as:

a) Segment 1

b) Segment 2

c) Segment 3

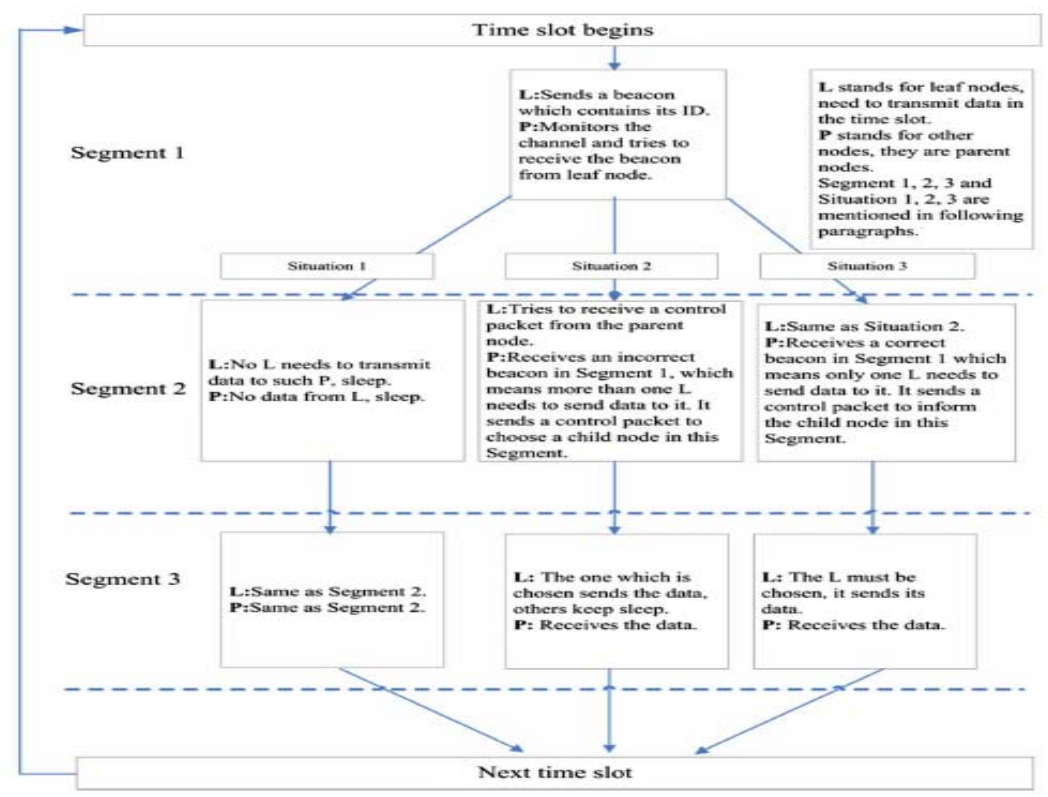

Figure 1. Process of the permitted leaf nodes send their data to their parent nodes

Segment 1:

In the first segment we use to check whether there is communication interface between the child nodes to parent node. The child node sends its ID to its parent node during that time.[6] At this time three situations occur so they divide the parent nodes into three kinds. In the First situation if there is no leaf node to parent node then there is nothing to send leaf node. In the Second situation if the parent node has more than the one leaf node then after the transmission of data the parent node gets the incorrect ID .from the leaf nodes means duplication occurs.[10] In the third situation if the parent node has only one leaf node then it receives the correct ID from the leaf node. These situation depends on the segment 2.

Segment 2:

The three situation can depend on the Segment 2. So in the first situation if the parent nodes turns into sleep mode until next time slot begins. For the Second situation to the child nodes a control packet can be sent from the parent nodes and this control packet chooses one of its child node to transmit the data in the next segment. For the third situation, a control packet received from the parent node and tells to leaf node to transmit the data in the next segments. In this segment the leaf nodes which can confirmed the data before it transmits [8].

Segment 3:

The permitted leaf nodes send their data to their parent nodes, while other leaf nodes in sleep mode. The process can be shown in the Figure 1 [10], [11].

Here the each node needs to generate and transmit a DATA PAK in each round then the node may exhaust its energy and die. If any node die then there will be change in the topography is more [4]. So to overcome it the nodes which die before it informs to neighbor nodes that particular nodes is dying. In each time slot, the nodes whose energy is going to be exhausted will compute a random delay which makes only onenodebroadcastin this time slot. When the delay is ended, these nodes are trying to broadcast a packet to 
the whole network. While all other nodes are monitoring the channel, they will receive this packet and perform an ID check [11], [12]. Then they modify their tables. If consider another case the BS collects the Initial EL and the co-ordinates information of all the sensors. Once the routing tree is built, the energy consumption of each sensor node in this round can be calculated by BS, thus the information needed for calculating the topology for the next round can be known in advance and can be shown below Figure 2. However, because WSN may be deployed in an unfriendly environment, the actual EL of each sensor node may be different from the EL calculated by BS. To cope with this problem, each sensor node calculates its EL and detects its actual residual energy in each round, So we define the calculated EL \& EL1. The sensor nodes generates the error flags and packs of data of residual energy into the DATA_PAK, which needs to be send to Base station [2], [8].

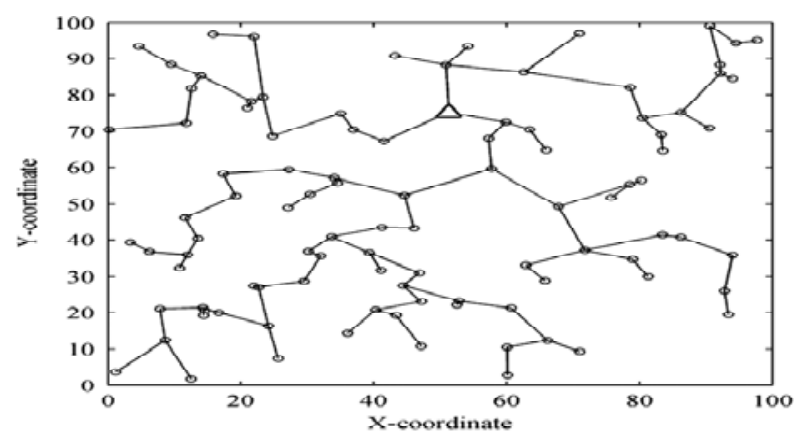

Figure 2. Routing tree generated by TEBRSO for 100 nodes

\section{CONCLUSION}

In this paper we introduced a protocol for better routing and the less usage of network life time. Here we compared TEBRSO protocol with other protocol like LEACH, PEGASIS, HEED etc. The TEBRSO is the self-organized protocol, it uses only small amount of energy in each round to change the topography for balancing the consumption of energy. Here all the leaf nodes uses the same time slot for transmitting the data so that the transmission delay will be less when we compare with other protocols. If any node before it die that particular node sends the information to the neighbor nodes to build the new topography. The TEBRSO is $200 \%$ much more efficient than the LEACH and $300 \%$ more efficient to the PEGASIS protocol, it is $100 \%$ more efficient than the HEED. By using this protocol we can balance the network load. Even though GSTEB needs BS to compute the topography, which leads to an increase in energy waste and longer delay, this kind of energy waste and longer delay are acceptable when compared with the energy consumption and the time delay for data transmitting. The works extends to targeted region and also the deployment and to scheduling the nodes in various region like $\mathrm{i}, \mathrm{k}, \mathrm{j}$ regions etc.

\section{REFERENCES}

[1] I. F. Akyildiz, W. Su, Y. Sankarasubramaniam, and E. Cayirci, "Wireless sensor networks: A survey," Computer Netw.s, vol. 38, No. 4, pp. 393-422, 2002.

[2] S. Lindsey and C. Raghavendra, "Pegasis: Power-efficient gathering in sensor information systems," In Proc. IEEE Aerospace Conf., Vol. 3, pp. 1125-1130, 2002.

[3] H. O. Tan and I. Korpeoglu, "Power efficient data gathering and aggregation in wireless sensor networks," SIGMOD Rec., Vol. 32, No. 4, pp. 66-71, 2003.

[4] S. S. Satapathy and N. Sarma, "TREEPSI: Tree based energy efficient protocol for sensor information," in Proc. IFIP Int. Conf., pp. 11-13, 2006.

[5] O. Younis and S. Fahmy, "HEED: A hybrid, energy-efficient, distributed clustering approach for ad hoc sensor networks," IEEE Trans. Mobile Computing, Vol. 3, No. 4, pp. 660-669, 2004.

[6] R. Szewczyk, J. Polastre, A. Mainwaring, and D. Culler, "Lessons from sensor network expedition," in Proc. 1st European Workshop on Wireless Sensor Networks EWSN '04, Germany, 2004.

[7] W. Liang and Y. Liu, "Online data gathering for maximizing network lifetime in sensor networks," IEEE Trans Mobile Computing, Vol. 6, No. 1, pp. 2-11, 2007.

[8] J. H. Chang and L. Tassiulas, "Energy conserving routing in wireless ad hoc networks," in Proc. IEEE INFOCOM, 2000, vol. 1, pp. 22-31.

[9] G. Mankar and S. T. Bodkhe, "Traffic aware energy efficient routing protocol," in Proc. 3rd ICECT, 2011, Vol. 6, pp. 316-320. 
[10] N. Tabassum, Q. E. K. Mamun, and Y. Urano, "COSEN: A chain oriented sensor network for efficient data collection," in Proc. IEEE ITCC, pp. 262-267, 2006.

[11] W. R. Heinzelman, A. Chandrakasan, and H. Balakrishnan, "Energy efficient communication protocols for wireless micro sensor networks," in Proc. 33rd Hawaii Int. Conf. System Sci., pp. 3005-3014, 2000.

[12] W. B. Heinzelman, A. Chandrakasan, and H. Balakrishanan, "An application-specific protocol architecture for wireless micro sensor networks," IEEE Trans.Wireless Commun, Vol. 1, No. 4, pp. 660-670, 2002. 